\title{
Comparative genomics of Klebsiella michiganensis BD177 and related members of Klebsiella sp. reveal the symbiotic relationship with Bactrocera dorsalis
}

Zhaohui Cai, Qiongyu Guo, Zhichao Yao, Wenping Zheng, Junfei Xie, Shuai Bai and Hongyu Zhang*

\begin{abstract}
Background: Bactrocera dorsalis is a destructive polyphagous and highly invasive insect pest of tropical and subtropical species of fruit and vegetable crops. The sterile insect technique (SIT) has been used for decades to control insect pests of agricultural, veterinary, and human health importance. Irradiation of pupae in SIT can reduce the ecological fitness of the sterile insects. Our previous study has shown that a gut bacterial strain BD177 that could restore ecological fitness by promoting host food intake and metabolic activities.

Results: Using long-read sequence technologies, we assembled the complete genome of K. michiganensis BD177 strain. The complete genome of K. michiganensis BD177 comprises one circular chromosome and four plasmids with a GC content of 55.03\%. The pan-genome analysis was performed on 119 genomes (strain BD177 genome and 118 out of 128 published Klebsiella sp. genomes since ten were discarded). The pan-genome includes a total of 49305 gene clusters, a small number of 858 core genes, and a high number of accessory (10566) genes. Pangenome and average nucleotide identity (ANI) analysis showed that BD177 is more similar to the type strain $K$. michiganensis DSM2544, while away from the type strain K. oxytoca ATCC13182. Comparative genome analysis with $21 \mathrm{~K}$. oxytoca and $12 \mathrm{~K}$. michiganensis strains, identified 213 unique genes, several of them related to amino acid metabolism, metabolism of cofactors and vitamins, and xenobiotics biodegradation and metabolism in BD177 genome.
\end{abstract}

Conclusions: Phylogenomics analysis reclassified strain BD177 as a member of the species K. michiganensis. Comparative genome analysis suggested that K. michiganensis BD177 has the strain-specific ability to provide three essential amino acids (phenylalanine, tryptophan and methionine) and two vitamins B (folate and riboflavin) to $B$. dorsalis. The clear classification status of BD177 strain and identification of unique genetic characteristics may contribute to expanding our understanding of the symbiotic relationship of gut microbiota and B. dorsalis.

Keywords: Sterile insect technique, Gut microbiota, Probiotics, Phylogenomics classification

\footnotetext{
* Correspondence: hongyu.zhang@mail.hzau.edu.cn

State Key Laboratory of Agricultural Microbiology, China-Australia Joint Research Centre for Horticultural and Urban Pests, Institute of Urban and Horticultural Entomology, College of Plant Science and Technology, Huazhong Agricultural University, Wuhan, People's Republic of China
} 


\section{Background}

Bactrocera dorsalis (Hendel) (Diptera: Tephritidae) is a destructive polyphagous and highly invasive insect pest of tropical and subtropical species of fruit and vegetable crops. While insecticides have been used to control this pest, insecticide resistance, and environmental pollution by chemical pesticides have been severely limiting this type of control [1]. Moreover, B. dorsalis has a powerful biological invasion ability. It is its invasion, spread, and establishment in sub-Saharan Africa and has caused about $\$ 2$ billion in economic losses in the horticultural export markets of Africa [2]. The current movement of B. dorsalis into Central China, without any apparent intense selective pressure, must pose a deep concern for other temperate regions of the world, especially Europe and North America [3].

The sterile insect technique (SIT) has been used for decades to control insect pests of agricultural, veterinary and human health importance $[4,5]$. Compared with insecticide control strategies, SIT has several attractive features, including species specificity and environment friendliness. Ionizing irradiation was used to sterilize insects, and these insects were subsequently handled, transported, and released in the field, ideally only males [6]. Thus, SIT can be an alternate strategy for the management of B. dorsalis. Previously, SIT has been used to control pest fruit fly species, including Ceratitis capitata [7], B. tryoni [8], B. cucurbitae [9] and B. dorsalis [10]. However, SIT may have some limitations related to the ecological fitness of sterile male adult flies due to domestication, mass-rearing, irradiation, and handling [11]. These procedures also impact the tephritid gut microbiome, with detrimental effects on physiology, behavior, and fitness $[12,13]$. Thus, the deleterious impact on the ecological fitness of the released insects has been one of the most considerable issues of SIT applications [11, 14].

Gut microbiota is strongly connected with the biology of the host and contributes to its health [15]. Gut microbiota affects insects in several ways, such as aiding food digestion and detoxification [16], providing essential nutrients [17], and protecting against infectious pathogens [18]. Much recent research of Tephritids suggests that gut microbiota reduced larval development time [19, 20], increased pupal weight [21], larger males [22], improved male performance $[13,23]$, increased female fecundity [24], increased longevity [23, 25] and increased chilling resistance [26]. Mass rearing and irradiation processes affect the gut microbial community structure in the Tephritids [13, 23, 27, 28]. Compared to wild flies, the abundance and diversity of the major gut microbiota community Enterobacteriaceae in mass-reared irradiated flies are reduced, and the abundance of the minor members (e.g., Pseudomonas or Bacillaceae) increased [13, 23]. This disturbance of gut microbial homeostasis may be causally related to the competitiveness disadvantaged of sterile males. The above researches show that the manipulation of gut microbiota has great potential and can be introduced to SIT facilities to improve the efficiency of pest control. Gut bacteria can be used as probiotics to prevent pathogens, promote larval growth, and male performance during all the production stages of sterile male fly, from the egg to the released fly.

Gut symbiotic bacteria community of $B$. dorsalis has been investigated $[23,27,29]$. Enterobacteriaceae were the predominant family of different $B$. dorsalis populations and different developmental stages from laboratory-reared and field-collected samples [27, 29]. Our previous study found that irradiation causes a significant decrease in Enterobacteriaceae abundance of the sterile male fly [23]. We succeed in isolating a gut bacterial strain BD177 (a member of the Enterobacteriaceae family) that can improve the mating performance, flight capacity, and longevity of sterile males by promoting host food intake and metabolic activities [23]. However, the probiotic mechanism remains to be further investigated. Therefore, the genomic characteristics of BD177 may contribute to an understanding of the symbionthost interaction and its relation to $B$. dorsalis fitness. The here presented study aims to elucidate the genomic basis of strain BD177 its beneficial impacts on the sterile males of $B$. dorsalis. An insight into strain BD177 genome feature helps us make better use of the probiotics or manipulation of the gut microbiota as an important strategy to improve the production of high performing B. dorsalis in SIT programs.

\section{Results}

\section{Assembly description and genome information of $K$. michiganensis BD177}

The genome of $K$. michiganensis BD177 was sequenced using the Illumina HiSeq and Pacbio technology. Raw reads $(\sim 1.3 \mathrm{Gbp})$ were processed to remove SMRT bell adapters, short and low-quality reads $(<80 \%$ accuracy) using SMRT Analysis version 2.3 (https://www.pacb. com/products-and-services/analytical-software/wholegenome-sequencing/). A total of 155,828 filtered reads (average length, $8.9 \mathrm{~Kb}$ ) were used for de novo assembly using Celera Assembler [30], with self-correction of the PacBio reads. A total of $1091 \mathrm{Mb}$ paired-end Illumina reads screened from a 500-bp genomic library were mapped using SOAP [31] to the bacterial plasmid database for tracing the presence of any plasmid and filling the gaps. The de novo assembly resulted in five contigs, representing the $K$. michiganensis BD177 complete genome of $6,812,698$ bp with GC content $55.03 \%$ in a single chromosome and four plasmids. The annotated genome contains 6714 genes, 25rRNA genes, and 86 tRNA genes (Table 1). 
Table 1 Genome features of isolated strain BD177

\begin{tabular}{ll}
\hline Features & BD177 \\
\hline Genome Size (bp) & $6,812,698$ \\
Length of Chromosome (bp) & $6,150,416$ \\
Numberof plasmids & 4 \\
Length of Plasmid1 (bp) & 281,962 \\
Length of Plasmid2 (bp) & 191,962 \\
Length of Plasmid3 (bp) & 164,510 \\
Length of Plasmid4 (bp) & 23,848 \\
GC content (\%) & $55.03 \%$ \\
No. of 5 s rRNA & 9 \\
No. of 16 s rRNA & 8 \\
No. of 23 s rRNA & 8 \\
No. of tRNA & 86 \\
Genes & 6714 \\
\hline
\end{tabular}

16S rRNA phylogenetic analysis and phenotypic of isolated strain BD177

Maximum parsimony based and maximum likelihood phylogenetic trees using the sequence that encodes for the 16S rRNA gene showed that the closest species to BD177 is $K$. michiganensis and $K$. oxytica (Additional file 1: Fig. S1, Additional file 2: Table S1). Evolutionary divergence distance of $16 \mathrm{~S}$ rRNA gene sequence between BD177 and $K$. michiganensis E718 was minimum value (BD177 vs $K$. michiganensis E718: 0.0079) among all sequences. Biochemical tests were performed on BD177 by API20E to confirm its position in the $K$. oxytoca and $K$. michiganensis. Biochemical characteristics of $K$. oxytoca ATCC13182(T)(=NCTC13727) and $K$. michiganensis W14(T)(= DSM2544) were obtained from BacDive Webservices [32]. Compared with $K$. oxytoca ATCC13182(T) and K. michiganensis W14(T), BD177 is the same with W14 in the $\beta$-galactosidase enzyme positive and urease negative, same with ATCC13182(T) in arginine dihydrolase positive. However, BD177 does not have the utilization ability of citrate as only carbon source, different from both type strains (Table 2).

Table 2 Phenotypic profiles of the isolated strain BD177

\begin{tabular}{|c|c|c|c|}
\hline Characteristics & BD177 & Klebsiella michiganensis W14(T) & Klebsiella oxytoca ATCC13182(T) \\
\hline ONPG & + & + & - \\
\hline ADH & + & - & + \\
\hline LDC & + & + & + \\
\hline ODC & - & - & - \\
\hline $\mathrm{CIT}$ & - & + & + \\
\hline $\mathrm{H} 2 \mathrm{~S}$ & - & - & - \\
\hline URE & - & - & + \\
\hline TDA & - & - & - \\
\hline IND & + & + & + \\
\hline VP & + & + & + \\
\hline GEL & - & - & - \\
\hline GLU & + & + & + \\
\hline MAN & + & + & + \\
\hline INO & + & + & + \\
\hline SOR & + & + & + \\
\hline RHA & + & + & + \\
\hline SAC & + & + & + \\
\hline MEL & + & + & + \\
\hline AMY & + & + & + \\
\hline ARA & + & + & + \\
\hline OX & - & - & - \\
\hline
\end{tabular}

ONPG $\beta$-galactosidase enzyme; $A D H$ arginine dihydrolase; $L D C$ lysine decarboxylase; $O D C$ ornithine decarboxylase; CIT utilization of citrate as only carbon source; $\mathrm{H} 2 \mathrm{~S}$ production of hydrogen sulfide; URE urease; TDA tryptophan deaminase; IND tryptophanase; VP acetoin produced from glucose; GEL gelatinase; GLU fermentation of glucose; MAN fermentation of mannose; INO fermentation of inositol; SOR fermentation of sorbitol; RHA fermentation of rhamnose; SAC fermentation of sucrose; MEL fermentation of melibiose; AMY fermentation of amygdalin; ARA fermentation of arabinose; OX cytochrome-c oxidase; Result: +, positive; -, negative 


\section{Pan-genome analysis}

In this study, we considered the 128 publicly available genome assemblies for the Klebsiella sp. (Additional file 3: Table S2). Of these genomes, 26 were originally annotated as $K$. aerogenes, 13 were $K$. michiganensis, 27 were K. oxytoca, and 15 were $K$. pneumoniae. 25 were $K$. quasipneumoniae, 1 was $K$. quasivariicola, 21 were $K$. variicola. The type strain genome of each species is included in these genomes. These genome assemblies were passed strict quality control (N75 values of $>10,000 \mathrm{bp},<500$ undetermined bases per 100,000 bases, $>90 \%$ completeness and $<5 \%$ contamination) by quast and checkm (Additional file 4: Table S3). This resulted in a total of 118 Klebsiella sp. strains studied, where ten low-quality genome assemblies were discarded. The GC contents of the species $K$. michiganensis, $K$. oxytoca, $K$. pneumoniae, $K$. variicola, and $K$. quasipneumoniae showed low intraspecies variation, with respective average values of 55.78 , 55.22, 57.25, 57.18 and 57.62\% (Fig. 1a), whereas larger interspecies diversity was observed. In contrast, $K$. aerogenes genomes can be divided into two groups, a large group showing a GC content in the range of that of Klebsiella aerogenes (54.62 to 55.16\%) and a small group of two genomes (57.02 and 57.16\%) showing a much greater GC content, similar to that of the K. pneumoniae, K.variicola and K. quasipneumoniae genomes (respective average values about 57\%). Based on GC content of the genome, K. pneumoniae, K.variicola, K. quasivariicola, and $K$. quasipneumoniae were considered as a high GC group content, and $K$. michiganensis and $K$. oxytoca were considered as a low GC content group. The genome sequence of our new isolate, which we designated $K$. michiganensis BD177 with $55.03 \%$ GC content (Table 1), shows a similar to that of the low GC content Klebsiella sp. group (Fig. 1a).

The pan-genome shape of the 119 analyzed Klebsiella $s p$. genomes is presented in Fig. 1b. Hard core genes are found in $>99 \%$ genomes, soft core genes are found in 95-99\% of genomes, shell genes are found in 15-95\%, while cloud genes are present in less than $15 \%$ of genomes. A total of 49,305 gene clusters were found, 858 of which comprised the core genome (1.74\%), 10,566 the accessory genome (21.43\%), and 37,795 (76.66\%) the cloud genome (Fig. 1b). Comparative genomic analysis evidenced that the 119 Klebsiella sp. pangenome can be considered as "open" since nearly 25 new genes are continuously added for each additional genome considered (Additional file 5: Fig. S2). To study the genetic relatedness of the genomic assemblies, we constructed a phylogenetic tree of the 119 Klebsiella sp. strains by using the presence and absence of core and accessory genes from pan-genome analysis (Fig. 2). The tree structure reveals six separate clades within 119 analyzed Klebsiella sp. genomes (Fig. 2). From this phylogenetic tree, type strain genomes originally annotated $K$. aerogenes, $K$. michiganensis, K. oxytoca, K. pneumoniae, K.variicola, and $K$. a

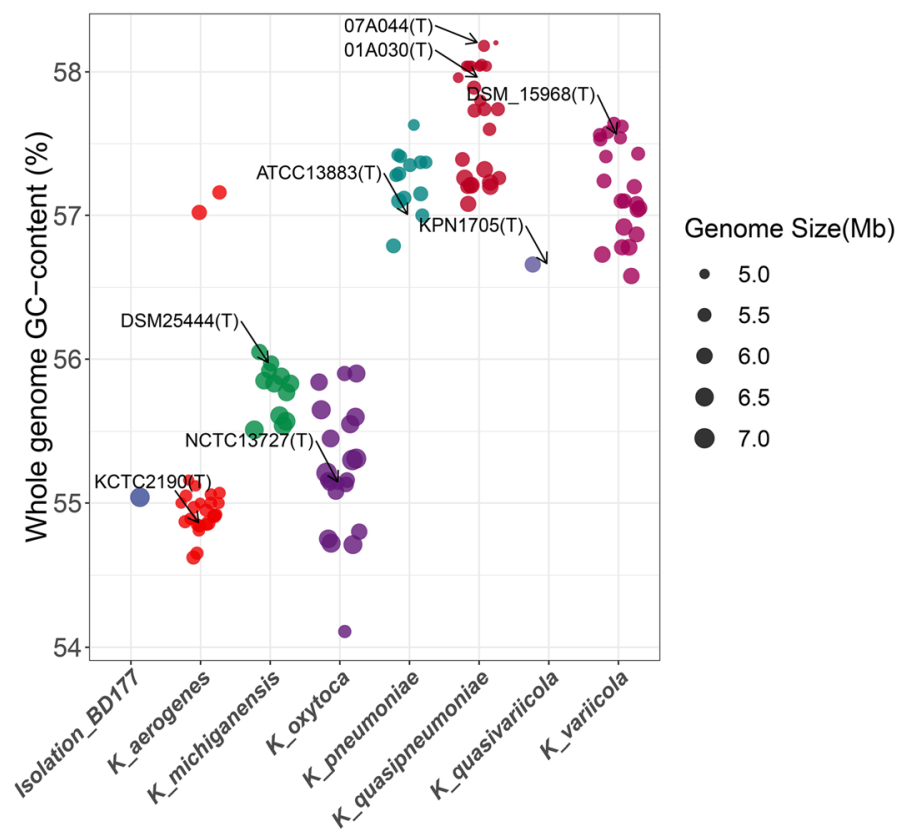

b

Pie chart of the 119 analyzed Klebsiella sp. genomes

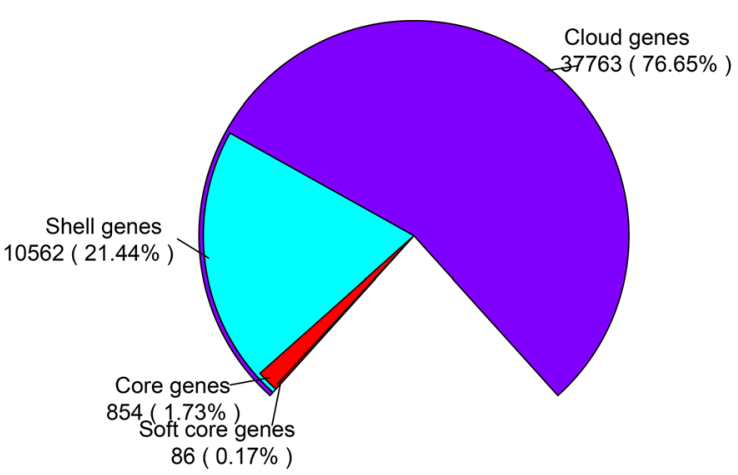

Fig. 1 Pan-genome analysis of 119 genomes in this study. Genomes are grouped according to their species annotation in the NCBI database (except for isolate K. michiganensis BD177). a GC content of all genomes is grouped according to their species annotation in the NCBI database. $\mathbf{b}$ pie chart of the 119 analyzed Klebsiella sp. genomes. The pan-genome pie chart showing gene content visualized with the use of Roary software 


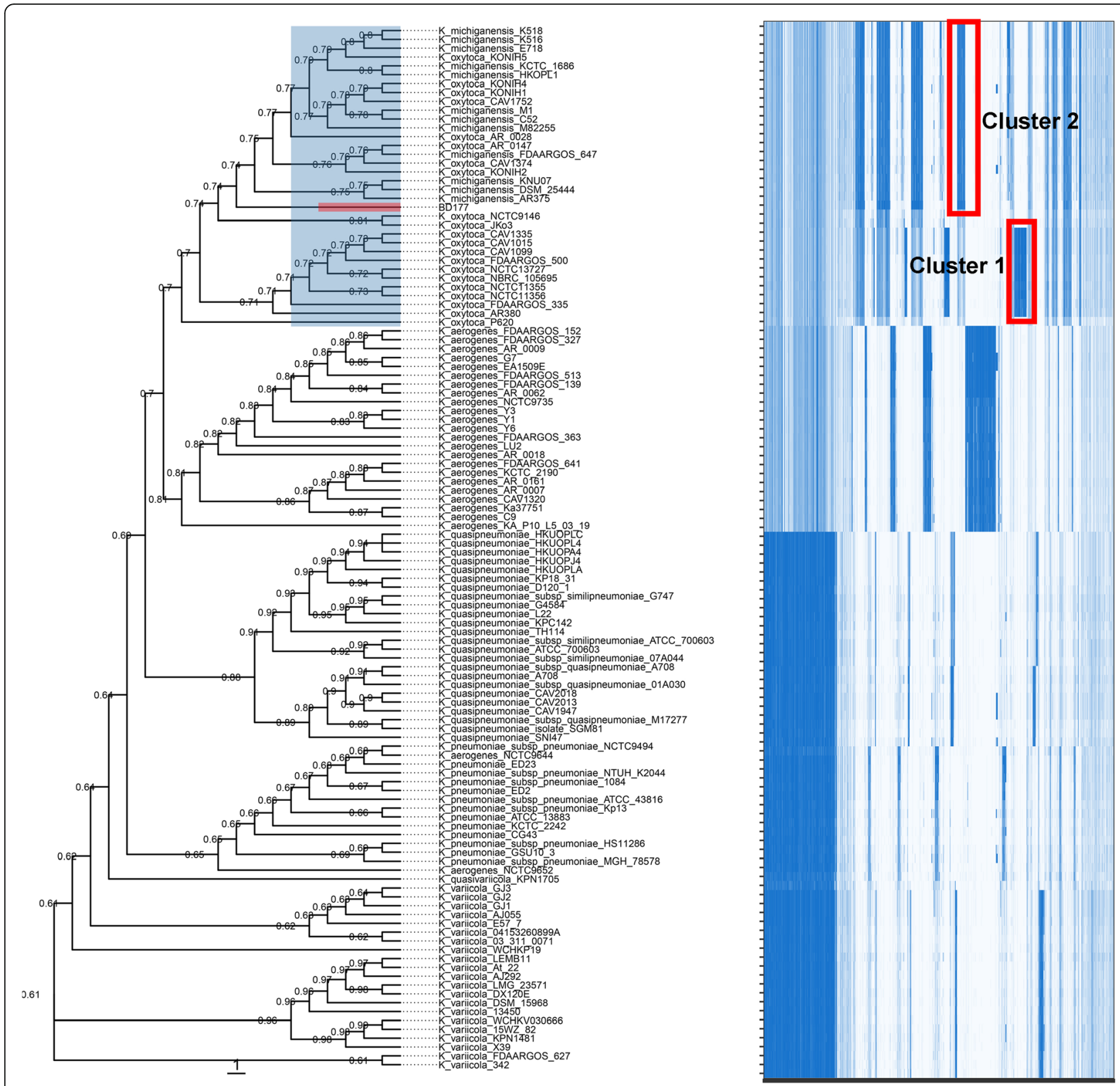

Fig. 2 Phylogenomics analysis of BD177 based on pan-genome. A heatmap was indicating gene presence (dark blue) or absence (light blue) in each of the 119 strains. A phylogeny built based on the presence and absence of core and accessory genes is shown on the left, and the strain names are indicated on the right

quasipneumoniae in the NCBI database were divided into six different clusters. Some non-type strain genomes originally annotated as $K$. oxytoca in the NCBI database are clustered in type strain $K$. michiganensis DSM25444 clade. The $K$. oxytoca group, including type strain $K$. oxytoca NCTC13727, have the unique gene cluster 1 (Fig. 2). K. michiganensis group, including type strain $K$. michiganensis DSM25444, has the unique cluster 2 (Fig. 2 ). Genes cluster 1 and cluster 2 based on unique presence genes from the pan-genome analysis can distinguish between non-type strain $K$. michiganensis and $K$. oxytoca (Fig. 2). However, our new isolated BD177 is clustered in type strain K. michiganensis clade (Fig. 2).

In the current era of high-throughput sequencing with easy access to bacterial genomes, average nucleotide identity (ANI) of genome-wide comparison has been recommended as a standard method to improve the accuracy of taxonomic identification of prokaryotic genomes [33]. Figure 3a shows the ANIm (ANI calculated by using a MUMmer3 [34] implementation) between all 119 Klebsiella sp. genomes. The distance metrics support the grouping of the genomes in the six clades 
a

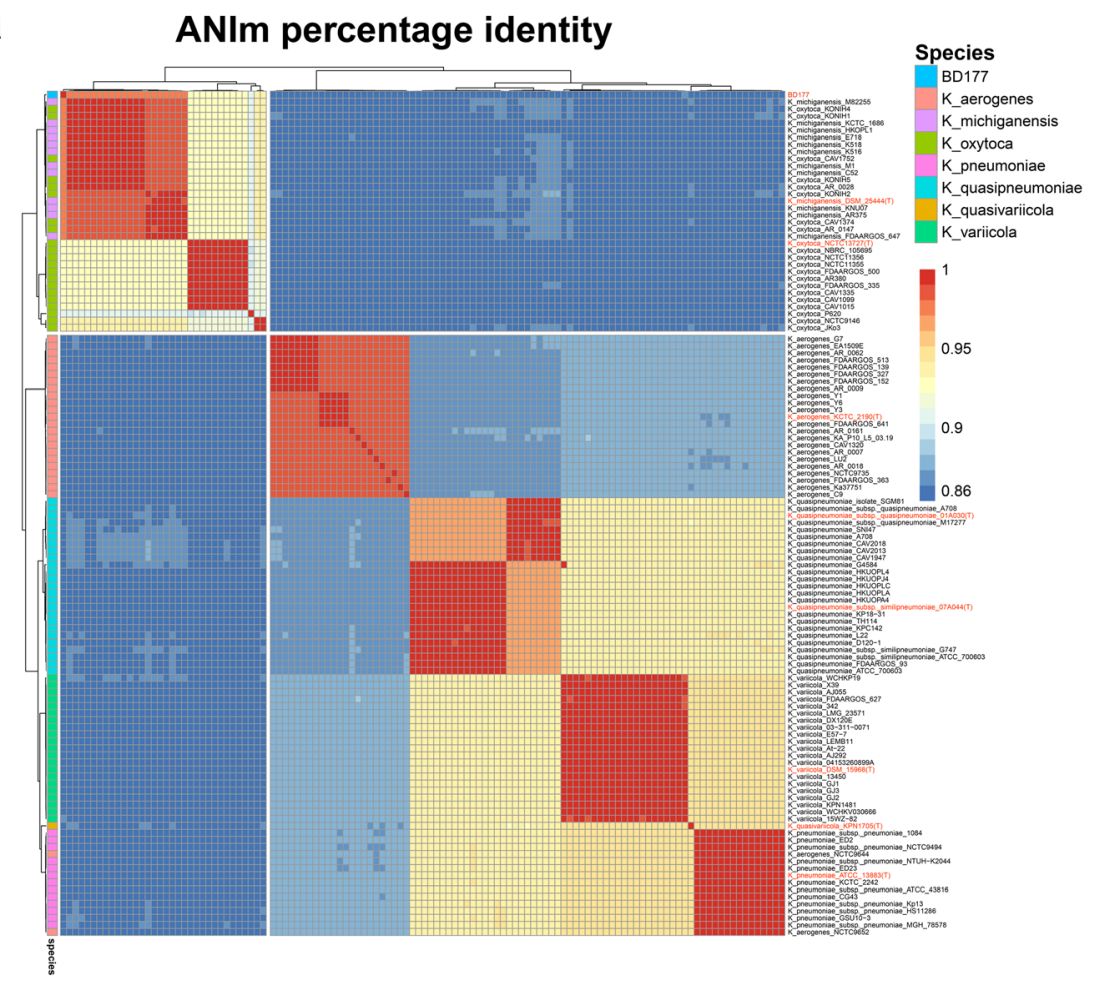

b

ANIm percentage identity

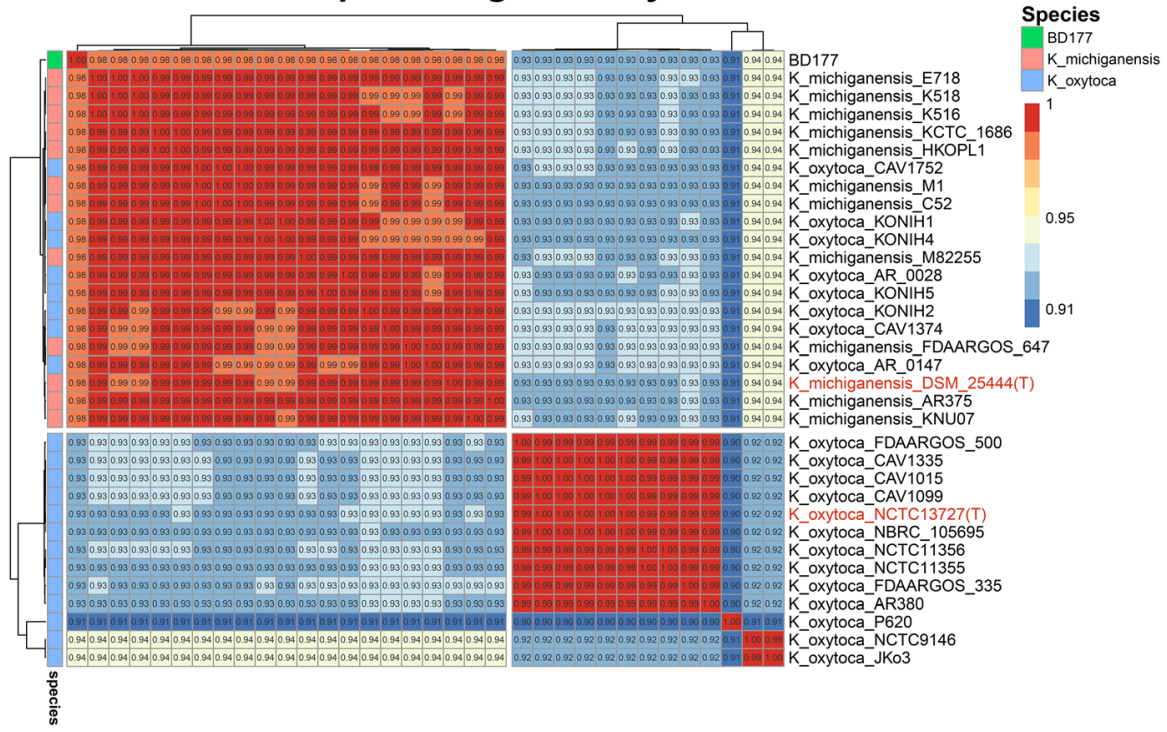

Fig. 3 Pairwise ANIm values for genomes. a Heatmap and hierarchal clustering of average nucleotide identity (ANI) values between BD177 and 118 Klebsiella sp. strains. See supplement for a table with ANI values (Additional file 7: Table S4). b Pairwise ANIm values for genomes. Heatmap and hierarchal clustering of average nucleotide identity (ANI) scores between BD177, 21 K. oxytoca and 12 K. michiganensis strains

defined by the phylogenetic tree in Fig. 2. For species delimitation, Ciufo et al. [35] advise the use of an ANI cutoff of $96 \%$ to define species boundaries. When comparing genomes belonging to different Klebsiella species, we observed ANI values of $\leq 94.8 \%$ (Fig. 3b and Additional file 6: Table S4), indicating that each clade consists of one species(expect for
K.oxytoca P620, K. oxytoca NCTC9146, and K. oxytoca JKo3) that are distinct from the other clades (Fig. 3b). The average nucleotide identity (ANI) value between BD177 and type strain $K$. michiganensis DSM25444 was $98 \%$ more than the cutoff of $96 \%$ (Fig. 3b), while between BD177 and type strain $K$. oxytoca NCTC13727 was 93\%. 
Genetic potential of BD177 for a symbiotic relationship Based on the pan-genome analysis of 119 Klebsiella sp. strains, we focused on the genomic relatedness of $K$. michiganensis BD177, $21 \mathrm{~K}$. oxytoca, and $12 \mathrm{~K}$. michiganensis strains. In total, 201,774 genes were present in their genomes, with an average number of 5934 genes per genome. These genes were clustered into 9643 orthogroups by OrthoFinder [36], where an orthogroup is defined as the group of genes descended from a single gene in the most recent common ancestor of a group of species. Of these orthogroups, 3833 were identified as core orthogroups, and 3351 were identified as singlecopy orthogroups (Additional file 7: Table S5). In $K$. michiganensis BD177, 213 strain-specific orthogroups were identified. Mapping of these orthogroups to the eggNOG database (v4.5) [37] revealed a unique in the predicted functional capacity (Additional file 8: Table S6). The metabolic features of $K$. michiganensis BD177 were also investigated by cumulatively mapping the unique functional KEGG genes of the $K$. michiganensis BD177 to the KEGG pathways (Fig. 4). The KEGG pathway analysis showed that BD177 harbored complete phenylalanine, tyrosine, and tryptophan biosynthesis pathways (KEGG genes K04518, K04093, K11646), and Cysteine and methionine metabolism pathways (KEGG genes K13060). The KEGG pathway analysis also showed that the BD177 strains harbored Metabolism of cofactors and vitamins pathway for riboflavin metabolism (KEGG genes K19286) and folate biosynthesis (KEGG genes K06920). In addition, BD177 strains harbored genes of the xenobiotics biodegradation and metabolism pathway (KEGG genes K01061 and K05797) for chlorocyclohexane and chlorobenzene degradation, fluorobenzoate degradation, and toluene degradation.

\section{Discussion}

Klebsiella sp. strains are among the dominant symbiotic bacteria in the gut of Tephritidae, with the ability to play diverse roles [11]. In this study, we describe the complete genome sequence of $K$. michichanensis BD177, isolated from wild adult $B$. dorsalis. We found that the taxonomic status of the $B$. dorsalis gut bacterial strain BD177 could not be identified only based on the $16 \mathrm{~S}$ rRNA gene phylogenetic tree and biochemical analysis of the strain. Hence, we used the Illumina HiSeq and Pacbio technology to assemble a complete $6.8 \mathrm{Mbp}$ length genome of $K$. michiganensis BD177. Using the genome-wide information, we identified the taxonomic status and strain-specific genome features of the BD177

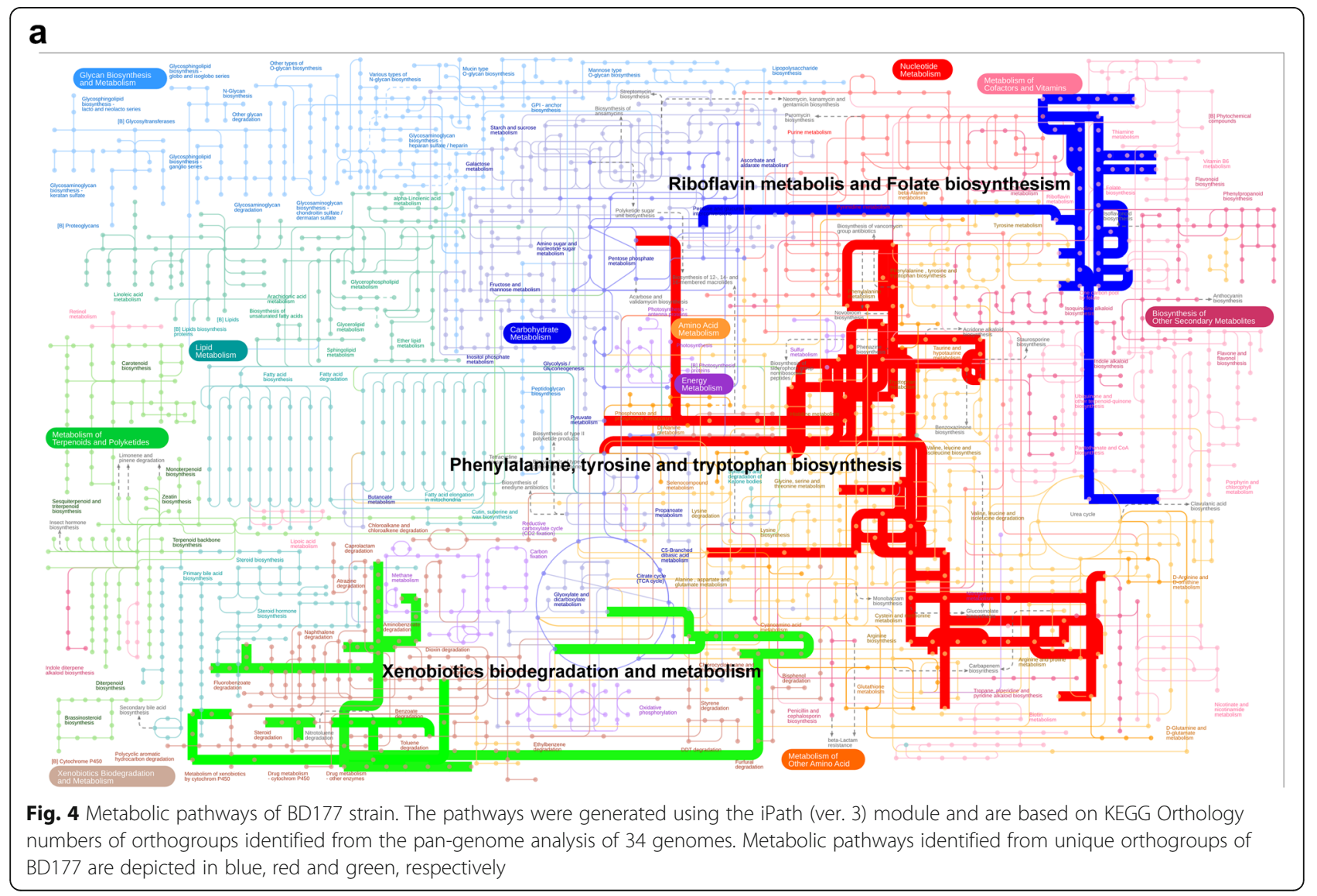


strain to explore the symbiotic relationship with $B$. dorsalis.

The phylogenetic analysis of the $16 \mathrm{~S}$ rRNA gene shows that the closest species to BD177 is $K$. oxytoca and $K$. michiganensis (Fig. S1). Bootstrap values were more than $70 \%$, which corresponds to a probability of $\geq 95 \%$ that the corresponding clade is real [38]. In addition, the biochemical indicators of the BD177 strain are not the same as the type strain of $K$. oxytoca and $K$. michiganensis(Table 2). Thus, the species status of the strain BD177 is still unclear. The 16S rRNA gene is often used as a putative marker for species circumscription, but the conservative nature of the gene did not show enough resolution on such a taxonomic scale [33]. Phenotypic properties can be unstable at times, and expression can be dependent upon changes in environmental conditions, e.g., growth substrate, temperature, and $\mathrm{pH}$ levels [39]. Furthermore, biochemical properties do not accurately reflect the entire extent of the genomic complexity of a given species [39].

As whole-genome sequencing has become more widely accessible due to the introduction of costeffective high-throughput DNA sequencing technology, it is evident that genome sequence similarities have been developed to be a routine taxonomic parameter. We compared the genome feature of BD177 with 118 currently available high-quality genomic assemblies of the Klebsiella sp., which comprises the species $K$. aerogenes, $K$. michiganensis, $K$. oxytoca, K. pneumoniae, $K$. variicola, and $K$. quasipneumoniae. Here we clarified the classification status of $K$. michiganensis BD177, compared with the six taxonomic clades on the basis of (i) differences in whole-genome GC content (Fig. 1a), (ii) a phylogenetic tree constructed on the presence and absence of core and accessory genes from pan-genome analysis (Fig. 2), and (iii) pairwise ANI (Fig. 3). All 118 Klebsiella sp. genomes were divided into a low GC content group (including $K$. aerogenes, $K$. michiganensis and $K$. oxytoca species) and a high GC content group (including $K$. pneumoniae, $K$. variicola and $K$. quasipneumoniae species). Strain BD177 with $55.03 \%$ GC content belongs to the low GC genome group. The GC content of complex microbial communities seems to be globally and actively influenced by the environment. Similar environments tend to have similar GC-content patterns [40]. A phylogenetic tree constructed on the presence and absence of core and accessory genes confirms the position of strain BD177 within the Klebsiella sp. strains. The $K$. michiganensis group, including type strain $K$. michiganensis DSM25444, have the unique gene cluster 2. K. oxytoca group, including type strain $K$. oxytoca NCTC13727, has the unique cluster 1 . Non-type strains $K$. michiganensis and $K$. oxytoca are distinguishable based on genes cluster 1 and cluster 2 from the pan- genome analysis. Whole-genome sequence data as a basis for taxonomic assignment display greater discriminatory power than $16 \mathrm{~S}$ rRNA gene sequence analysis alone [41]. In addition, pairwise genome comparison metrics such as average nucleotide identity (ANI) is also used as a reliable method to verify taxonomic identities in prokaryotic genomes, for both complete and draft assemblies [33]. Based on average nucleotide identity (ANI) value with the type strain $K$. michiganensis and $K$. oxytoca similarity, BD177 belongs to $K$. michiganensis species rather than $K$. oxytoca species (Fig. $3 \mathrm{~b}$ ). This result is consistent with the phylogenetic analysis base on the pan-genome. Strain BD177 belongs to $K$. michiganensis.

To explore potential probiotic of $K$. michiganensis BD177, an in-depth comparative genomics analysis of 34 genomes, including $21 \mathrm{~K}$. oxytoca, $12 \mathrm{~K}$. michiganensis and K. michiganensis BD177, was performed by Orthofinder. We found the 213 strain-specific orthogroups of the strain BD177 were identified from a total of 9643 orthogroups in comparative genomics analysis. Predicted functional capacity analysis of these orthogroups showed that these unique orthogroups include metabolic key enzymes of amino acid, vitamins and xenobiotics. Of potential importance to the symbiosis of strain BD177 with the insect, the host is the bacterium's encoded ability to biosynthesize the phenylalanine, tyrosine, tryptophan, cysteine and methionine. Our previous research also showed that supplementation of K. michiganensis BD177 to sterile male $B$. dorsalis improved total free amino acid levels in hemolymph [23]. The obligate primary endosymbionts of many sap-feeding insects provide their hosts with essential amino acids [42-44]. The symbiotic fungi of Drosophila melanogaster promote amino acid harvest to rescue the lifespan of undernourished flies [45]. It suggested that K. michiganensis BD177 can provide amino acids, especially essential amino acids such as phenylalanine, tryptophan and methionine, to the $B$. dorsalis.

Additionally, K. michiganensis BD177 was found to encode the ability to biosynthesize the $\mathrm{B}$ vitamins riboflavin (B2) and folate (B9). In humans, gut microbiota can synthesize and supply vitamins B to their hosts, which lack the biosynthetic capacity for most vitamins [46]. Recent studies have implicated the Drosophila microbiota in supplying folate [47], riboflavin [48] and thiamine [49]. The riboflavin and folate biosynthesize ability of $K$. michiganensis BD177 suggests these B-vitamins may be of particular importance, especially in adult life stages fed on undernourished nectar and dew [50]. In D. melanogaster, Acetobacter pomorum provides thiamine to its host to promote larval development [49]. Folate (B9) biosynthesis of Wigglesworthia glossinidia plays a role in Glossina morsitans maturation and reproduction [51]. 
Our previous research also showed that $K$. michiganensis BD177 improved the mating competitiveness and lifespan of sterile male $B$. dorsalis [23]. Additionally, the recent study reported that $K$. oxytoca could affect the foraging decision [52] and mate-selection [53] of B. dorsalis. It is suggested that riboflavin and folate synthetic ability of $K$. michiganensis BD177 may contribute to the sexual performance and lifespan of $B$. dorsalis.

Compared with other 33 Klebsiella sp. genomes, some strain-specific genes from xenobiotics biodegradation and metabolism pathway were also identified in the $K$. michiganensis BD177 genome. Recent research shows that gut bacteria of insects significantly contribute to resistance against xenobiotics, including phytotoxins and pesticides [54]. Pseudomonas fulva can assist with digestion and detoxification of caffeine as alkaloid allelochemical in the coffee berry borer [16]. Gut symbiont Burkholderia of Riptortus pedestrians has gained the ability to hydrolyze insecticide fenitrothion [55]. Gut symbiont Citrobacter sp. can degrade trichlorphon and conferred host insecticide resistance in $B$. dorsalis [56]. Interestingly, gut symbiont Lactobacillus plantarum of D. melanogaster can significantly enhance the toxicity of insecticide chlorpyrifos [57]. It is hypothesized that $K$. michiganensis BD177 may play a role in insect resistance against pesticides in the wild field. However, the potential mechanisms of resistance or sensitivity of insect to insecticide are unclear.

\section{Conclusions}

Supplement of gut symbiotic bacteria as probiotic to the larval or adult diet are encouraging for their potential application in SIT programs to produce high-quality insects. However, understanding of the bacterial probiotic mechanism is important for the selection and application of different insect species probiotics. Using longread sequence technologies, we assembled the complete genome of $K$. michiganensis BD177 strain. The comparison of the genome sequence against other Klebsiella species showed a percentage of genes that are unique to the BD177 strain, including metabolic key enzymes of amino acid, vitamins, and xenobiotics that could play an important role in the resistance and fitness of $B$. dorsalis. These findings extend our previous work [23] to improve the understanding of the relationship between gut bacteria and $B$. dorsalis. In the future, we can engineer the gut bacteria strain symbionts by strengthening the probiotic genetic elements of bacteria. It will improve the application efficiency of gut microbiota in pest management programs incorporating SIT.

\section{Methods}

$16 \mathrm{~S}$ rRNA gene analysis and biochemical characterization The Klebsiella michiganensis BD177 reported in this study was isolated in a previous study from the gut of $B$. dorsalis male adult, collected from the Institute of Urban and Horticultural Pests of Huazhong Agricultural University [23]. The bacterial DNA was extracted with the HiPure Bacterial DNA Kit (Magen) following the protocol for Gram-negative bacteria and used for the amplification of $16 \mathrm{~S}$ rRNA gene using the primers $27 \mathrm{~F}$ (5'GTTTGATCCTGGCTCAG-3') and 1492R (5'-GGTT ACCTTGTTACGACTT-3') [16]. Subsequently, the $1.4 \mathrm{~kb}$ PCR product was purified using a PCR purification kit (Axygen) and was subjected to bidirectional Sanger sequencing. The 16S rRNA gene sequence of strain BD177 was compared with the reference sequences using the "identify" tool of EzBioCloud database for the taxonomic assignment [58]. The similarity of the 16S rRNA gene between strain BD177 to Klebsiella michiganensis type strain W14 and Klebsiella oxytoca type strain JCM 1665 was 99.25 and $99.15 \%$, respectively. In addition, 16S rRNA gene sequences longer than 1300 nucleotides of type strains $K$. oxytoca, $K$. michiganensis, K. pneumonia, K. quasipneumoniae, K. aerogenes, Klebsiella variicola, and Pseudomonas aeruginosa were downloaded from EzBioCoud database [58]. Alignments of the sequences were performed using the MUSCLE software [59]. A neighbor-joining phylogenetic tree based on sequences of the 16S rRNA gene was constructed with the Molecular Evolutionary Genetics Analysis (MEGA X) [60], using the Kimura 2-parameter model with 1000 bootstrap replicates. The maximum parsimony and maximum likelihood trees with the inclusion of outgroup were constructed by MEGA X. Distance matrix of evolutionary divergence between $16 \mathrm{~s}$ rRNA gene sequences was estimated by MEGA X. Strain BD177 was biochemically confirmed by using the API 20E system according to the manufacturer's instructions, which is a biochemical panel for identification and differentiation of members of the family Enterobacteriaceae (bioMerieux Inc., Hazelwood, MO) [61].

\section{DNA isolation and genome sequencing}

K. michiganensis BD177 strain was grown on LB, with incubation at $37^{\circ} \mathrm{C}$ in aerobic conditions. For DNA extraction, the strain was grown in $8 \mathrm{ml}$ of medium for overnight, followed by pelleting at $6000 \mathrm{~g}$ for $10 \mathrm{~min}$, and genomic DNA was obtained using E.Z.N.A. Bacterial DNA Kit (OMEGA). The bacterial DNA extraction was followed by quantity and quality estimation using a NanoDrop (Thermo Scientific) and also visualization of aliquots onto a $1.2 \%$ agarose gel stained with ethidium bromide to verify DNA integrity. Whole-genome sequencing of Klebsiella michiganensis BD177 was performed on the PacBio RS II platform and Illumina HiSeq 4000 platform at the Beijing Genomics Institute (BGI, Shenzhen, China). For Illumina sequencing, genomic DNA was sheared randomly to construct three 
read libraries with lengths 500 bp by a Bioruptor ultrasonicator (Diagenode, Denville, NJ, USA) and physicochemical methods. DNA was sequenced on a HiSeq sequencer (Illumina) with pair-end $125 \mathrm{bp}$ reads. Low-quality trimming and adapter removal for the Illumina reads was performed using Trimmomatic [62], resulting in a total of $1091 \mathrm{Mb}$ clean data. For Pacbio sequencing, the program pbdagcon (https://github.com/PacificBiosciences/pbdagcon) was used for self-correction. Then these reads were filtered using the RS_Subreads protocol (minimum subread length $=1 \mathrm{~kb}$, minimum polymerase read quality $=0.8$ ), resulting in a total of $1394 \mathrm{Mb}$ useable data (total number of subreads $=155,828$ reads, mean subread length $=8897$ bp, subread N50 = 11,669 bp).

\section{Genome assembly and annotation of K. michiganensis BD177}

Draft genomic unitigs, which are uncontested groups of fragments, were assembled using the Celera Assembler [30] against a high quality corrected circular consensus sequence subreads set. To improve the accuracy of the genome sequences, GATK (https://www.broadinstitute.org/ gatk/), and SOAP tool packages (SOAP2, SOAPsnp, SOAPindel) were used to make single-base corrections [31]. To trace the presence of any plasmid, the filtered Illumina reads were mapped using SOAP [31] to the bacterial plasmid database (last accessed 5 March 2018) [63].

Gene prediction was performed on the K. michiganensis BD177 genome assembly by glimmer3 [64] with Hidden Markov Models. tRNA, rRNA, and sRNAs recognition made use of tRNAscan-SE [65], RNAmmer [66] and the Rfam database [67]. The tandem repeats annotation was obtained using the Tandem Repeat Finder [68], and the minisatellite DNA and microsatellite DNA selected based on the number and length of repeat units. The Genomic Island Suite of Tools (GIST) used for genomics lands analysis [69] with IslandPath-DIOMB, SIGI-HMM, IslandPicker method. Prophage regions were predicted using the PHAge Search Tool (PHAST) webserver [70] and CRISPR identification using CRISPRFinder [71].

Seven databases, which are KEGG (Kyoto Encyclopedia of Genes and Genomes) [72], COG (Clusters of Orthologous Groups) [73], NR (Non-Redundant Protein Database databases) [74], Swiss-Prot [75], and GO (Gene Ontology) [76], TrEMBL [75], EggNOG [37] are used for general function annotation. A wholegenome BLAST search (E-value below 1e-5, minimal alignment length percentage above 40\%) was performed against the above seven databases. Virulence factors and resistance genes were identified based on the core dataset in VFDB (Virulence Factors of Pathogenic Bacteria) [77] and ARDB (Antibiotic Resistance Genes Database) database [78]. The molecular and biological information on genes of pathogen-host interactions were predicted by PHI-base [79]. Carbohydrate-active enzymes were predicted by the Carbohydrate-Active enZYmes Database [80]. Type III secretion system effector proteins were detected by EffectiveT3 [81]. Default settings were used in all software unless otherwise noted.

\section{Pan-genome analysis}

All complete genomic assemblies classified as $K$. oxytoca and $K$. michiganensis were downloaded from the NCBI database on 19 March 2019 with NCBI-GenomeDownload scripts (https://github.com/kblin/ncbi-genome-download). Genomic assemblies of $K$. pneumonia, $K$. quasipneumoniae, $K$. quasivariicola, $K$. aerogenes, and Klebsiella variicola type strains also were manually obtained from the NCBI database. The quality of the genomic assemblies was evaluated by QUAST [82] and CheckM [83]. Genomes with N75 values of $<10,000 \mathrm{bp}$, $>500$ undetermined bases per 100,000 bases, $<90 \%$ completeness, and $>5 \%$ contamination were discarded. The whole-genome GC content was calculated with QUAST [82]. All pairwise ANIm (ANI calculated by using a MUMmer3 implementation) values were calculated with the Python pyani package [34]. To avoid possible biases in the comparisons due to different annotation procedures, all the genomes were re-annotated using Prokka [84]. The pan-genome profile including core genes $(99 \%<=$ strains $<=100 \%)$, soft core genes $(95 \%<=$ strains $<99 \%)$, shell genes $(15 \%<=$ strains $<95 \%)$ and cloud genes $(0 \%<=$ strains $<15 \%)$ of 119 Klebsiella strains was inferred with Roary [85]. The generation of a 773,658 bp alignment of 858 single-copy core genes was performed with Roary [85]. The phylogenetic tree based on the presence and absence of accessory genes among Klebsiella genomes was constructed with FastTree [86] using the generalized time-reversible (GTR) models and the -slow, -boot 1000 option.

\section{Unique genes inference and analysis}

Orthogroups of BD177 and 33 Klebsiella sp. (K. michiganensis and $K$. oxytoca) genome assemblies were inferred with OrthoFinder [36]. All protein sequences were compared using a DIAMOND [87] all-against-all search with an E-value cutoff of $<1 \mathrm{e}-3$. A core orthogroup is defined as an orthogroup present in $95 \%$ of the genomes. The single-copy core gene, pan gene families, and core genome families were extracted from the OrthoFinder output file. "Unique" genes are genes that are only present in one strain and were unassigned to a specific orthogroup. Annotation of BD177 unique genes was performed by scanning against a hidden Markov model (HMM) database of eggNOG profile HMMs [37]. KEGG pathway information of BD177 unique orthogroups was visualized in iPath3.0 [88]. 


\section{Supplementary Information}

The online version contains supplementary material available at https://doi. org/10.1186/s12863-020-00945-0.

Additional file 1 Figure S1. Phylogenetic tree based on 165 rRNA gene sequences indicating the relationship between isolate BD177 with other type strains of the family Enterobacteriaceae, constructed using MUSCLE and MEGAX. (a) A maximum likelihood phylogenetic tree based on 165 rRNA gene sequences was assessed using bootstrap with 1000 replicates. Pseudomonas aeruginosa JCM5962 was selected as the outgroup. (b) A maximum parsimony phylogenetic tree based on $16 \mathrm{~S}$ rRNA gene sequences was assessed using bootstrap with 1000 replicates.

Additional file $\mathbf{2}$ Table S1. Distance matrix of evolutionary divergence between $16 \mathrm{~s}$ rRNA gene sequences. This distance matrix was obtained by a bootstrap procedure (1000 replicates) and the Kimura 2-parameter model.

Additional file $\mathbf{3}$ Table $\mathbf{S 2}$. RefSeq assembly accession numbers and strain name of the 128 Klebsiella sp. genomes from the NCBI database used in this study.

Additional file $\mathbf{4}$ Table S3. Overview of the various metrics employed to evaluate strain BD177 and 128 Klebsiella sp. genomes using QUAST and CheckM.

Additional file 5 Fig. S2. Diagram of conserved genes per number of genomes from the pan-genome analysis by Roary.

Additional file 6 Table S4. ANI values (\%) for each pairwise genome comparison of 119 genomes passed strict quality control.

Additional file $\mathbf{7}$ Table S5. General statistics about orthogroup sizes and the proportion of genes assigned to orthogroups by OrthoFinder

Additional file $\mathbf{8}$ Table S6. Annotate results of the unique orthogroups of BD177 from comparative genomics by eggNOGmapper.

\section{Abbreviations}

SIT: Sterile insect technique; bp: Base pair; kb: Kilo base pairs; Mb: Mega base pairs; Gbp: Giga base pairs; PCR: Polymerase chain reaction; LB: Luria Bertani; tRNA: Transfer ribonucleic acid; rRNA: Ribosomal ribonucleic acid; sRNAs: Small ribonucleic acid regulators; N50: The sequence length of the shortest contig at $50 \%$ of the total genome length; N75: The sequence length of the shortest contig at $75 \%$ of the total genome length; GC content: The percentage of guanine $(\mathrm{G})$ and cytosine $(C)$ in a certain fragment of DNA or RNA or for an entire genome; ANI: Average Nucleotide Identity.

\section{Acknowledgments}

The authors thank Hualin Liu, Ph.D., from the State Key Laboratory of Agricultural Microbiology for providing bioinformatics assistance.

\section{About this supplement}

This article has been published as part of BMC Genetics Volume 21 Supplement 2, 2020: Comparing rearing efficiency and competitiveness of sterile male strains produced by genetic, transgenic or symbiont-based technologies. The full contents of the supplement are available online at https://bmcgenet. biomedcentral.com/articles/supplements/volume-21-supplement-2.

\section{Authors' contributions}

H.Y.Z. conceived and designed the project., Z.H.C. designed and performed experiments and analyzed all data. Q.Y.G, Z.C.Y and S.B. performed isolation and identification of gut bacteria strains. Z.C.Y, W.P.Z and J.F.X analyzed the data of high-throughput sequencing. H.Y.Z. and Z.H.C. wrote the manuscript. All authors read and approved the final manuscript.

\section{Funding}

The design, collection and high-throughput sequencing of bacteria genome of the study were funded by the National Key R \& D Program of China (No. 2019YFD1002100), the National Natural Science Foundation of China (Nos. 31872931 and 31572008) and the earmarked fund for the China Agricultura Research System (No. CARS-26). Data analysis and computing resources is supported by the International Atomic Energy Agency research contact no. 18805 as part of the Coordinated Research Project "Comparing Rearing
Efficiency and Competitiveness of Sterile Male Strains Produced by Genetic, Transgenic or Symbiont-based Technologies" and the Fundamental Research Funds for the Central Universities (Nos. 2662019PY055 and 2662015PY129). Publication costs are funded by the Joint FAO/IAEA Division of Nuclear Techniques in Food and Agriculture, IAEA (CRP No.: D4.20.16) Vienna, Austria.

\section{Availability of data and materials}

The genome sequence data for the BD177 strain reported in this study can be retrieved from the National Center for Biotechnology Information (accession no. PRJNA602959). The other Klebsiella sp. strains genomes sequence analyzed in this study were downloaded from and are available in the GenBank database (https://www.ncbi.nlm.nih.gov/genbank/). The accession numbers of the 128 Klebsiella sp. genomes are shown in Additional file 3: Table S2.

Ethics approval and consent to participate

Not applicable.

\section{Consent for publication}

Not applicable.

\section{Competing interests}

The authors declare that they have no competing interests.

Published: 18 December 2020

\section{References}

1. Wei DD, He W, Lang N, Miao ZQ, Xiao LF, Dou W, Wang JJ. Recent research status of Bactrocera dorsalis: insights from resistance mechanisms and population structure. Arch Insect Biochem Physiol. 2019;102(3):e21601.

2. Ekesi S, De Meyer M, Mohamed SA, Virgilio M, Borgemeister C. Taxonomy, ecology, and management of native and exotic fruit fly species in Africa. Annu Rev Entomol. 2016:61:219-38.

3. Qin YJ, Krosch MN, Schutze MK, Zhang Y, Wang XX, Prabhakar CS, Susanto A, Hee AKW, Ekesi S, Badji K, et al. Population structure of a global agricultural invasive pest, Bactrocera dorsalis (Diptera: Tephritidae). Evol Appl. 2018;11(10):1990-2003.

4. Lees RS, Gilles JR, Hendrichs J, Vreysen MJ, Bourtzis K. Back to the future: the sterile insect technique against mosquito disease vectors. Curr Opin Insect Sci. 2015;10:156-62.

5. Klassen W, Curtis CF. History of the sterile insect technique. In: Dyck VA, Hendrichs J, Robinson AS, editors. Sterile insect technique principles and practice in area-Wide Integrated Pest Management. Dordrecht: Springer; 2005. p. 3-36.

6. Lance DR, McInnispp DO. Biological basis of the sterile insect technique. In: Dyck A, Hendrichs J, Robinson AS, editors. Sterile insect technique: principles and practice in area-wide integrated pest management. Dordrecht: Springer; 2005. p. 69-94.

7. Hendrichs J, Ortiz G, Liedo P, Schwarz A. Six years of successful medfly program in Mexico and Guatemala. Fruit Flies of Economic Importance. AA Balkema, Rotterdam, The Netherlands; 1983. p. 353-65.

8. Collins S, Weldon CW, Banos C, Taylor P. Optimizing irradiation dose for sterility induction and quality of Bactrocera tryoni. J Econ Entomol. 2009; 102(5):1791-800

9. Mau $R$, Jang $E$, Vargas $R$. The Hawaii fruit fly area-wide fruit fly pest management programme: Influence of partnership and a good education programme. In: Vreysen MJB Robinson AS Hendrichs J, editors. Area-Wide Control of Insect Pests: From Research to Field Implementation. Dordrecht: Springer; 2007. p. 671-83.

10. Orankanok W, Chinvinijkul S, Thanaphum S, Sitilob P, Enkerlin W. Area-wide integrated control of oriental fruit fly Bactrocera dorsalis and guava fruit fly Bactrocera correcta in Thailand. In: Vreysen MJB Robinson AS Hendrichs J, editors. Area-Wide Control of Insect Pests: From Research to Field Implementation. Dordrecht: Springer; 2007. p. 517-26.

11. Deutscher AT, Chapman TA, Shuttleworth LA, Riegler M, Reynolds OL. Tephritid-microbial interactions to enhance fruit fly performance in sterile insect technique programs. BMC Microbiol. 2019;19(Suppl 1):287.

12. Leftwich PT, Bolton M, Chapman T. Evolutionary biology and genetic techniques for insect control. Evol Appl. 2016;9(1):212-30. 
13. Ami EB, Yuval B, Jurkevitch E. Manipulation of the microbiota of mass-reared Mediterranean fruit flies Ceratitis capitata (Diptera: Tephritidae) improves sterile male sexual performance. ISME J. 2010;4(1):28-37.

14. Augustinos A, Targovska A, Cancio-Martinez E, Schorn E, Franz G, Cáceres C, Zacharopoulou A, Bourtzis K. Ceratitis capitata genetic sexing strains: laboratory evaluation of strains from mass-rearing facilities worldwide. Entomologia Experimentalis et Applicata 2017, 164(3):305-317.15. Douglas AE: multiorganismal insects: diversity and function of resident microorganisms. Annu Rev Entomol. 2015;60:17-34.

15. Douglas AE. Multiorganismal insects: diversity and function of resident microorganisms. Ann Rev Entomol. 2015;60:17-34.

16. Ceja-Navarro JA, Vega FE, Karaoz U, Hao Z, Jenkins S, Lim HC, Kosina P, Infante F, Northen TR, Brodie EL. Gut microbiota mediate caffeine detoxification in the primary insect pest of coffee. Nat Commun. 2015;6: 7618.

17. Shin SC, Kim S-H, You H, Kim B, Kim AC, Lee K-A, Yoon J-H, Ryu J-H, Lee WJ. Drosophila microbiome modulates host developmental and metabolic homeostasis via insulin signaling. Science. 2011;334(6056):670-4.

18. Mockler BK, Kwong WK, Moran NA, Koch H. Microbiome structure influences infection by the parasite Crithidia bombi in bumble bees. Appl Environ Microbiol. 2018;84(7):e02335-17.

19. Shuttleworth LA, Khan MAM, Osborne T, Collins D, Srivastava M, Reynolds $\mathrm{OL}$. A walk on the wild side: gut bacteria fed to mass-reared larvae of Queensland fruit fly [Bactrocera tryoni (Froggatt)] influence development. BMC Biotechnol. 2019;19(2):1-11.

20. Augustinos AA, Kyritsis GA, Papadopoulos NT, Abd-Alla AM, Cáceres C, Bourtzis K. Exploitation of the medfly gut microbiota for the enhancement of sterile insect technique: use of Enterobacter sp. in larval diet-based probiotic applications. PLoS One. 2015;10(9):e0136459.

21. Yao M, Zhang H, Cai P, Gu X, Wang D, Ji Q. Enhanced fitness of a Bactrocera cucurbitae genetic sexing strain based on the addition of gut-isolated probiotics (Enterobacter spec.) to the larval diet. Entomol Exp Applicata. 2017;162(2):197-203

22. Hamden $\mathrm{H}$, Guerfali MMS, Fadhl S, Saidi M, Chevrier C. Fitness improvement of mass-reared sterile males of Ceratitis capitata (Vienna 8 strain)(Diptera: Tephritidae) after gut enrichment with probiotics. J Econ Entomol. 2013; 106(2):641-7.

23. Cai Z, Yao Z, Li Y, Xi Z, Bourtzis K, Zhao Z, Bai S, Zhang H. Intestinal probiotics restore the ecological fitness decline of Bactrocera dorsalis by irradiation. Evol Appl. 2018;11(10):1946-63.

24. Sacchetti P, Ghiardi B, Granchietti A, Stefanini F, Belcari A. Development of probiotic diets for the olive fly: evaluation of their effects on fly longevity and fecundity. Ann Appl Biol. 2014;164(1):138-50.

25. Behar A, Yuval B, Jurkevitch E. Gut bacterial communities in the Mediterranean fruit fly (Ceratitis capitata) and their impact on host longevity. J Insect Physiol. 2008;54(9):1377-83.

26. Raza MF, Wang Y, Cai Z, Bai S, Yao Z, Awan UA, Zhang Z, Zheng W, Zhang H. Gut microbiota promotes host resistance to low-temperature stress by stimulating its arginine and proline metabolism pathway in adult Bactrocera dorsalis. PLoS Pathog. 2020;16(4):e1008441.

27. Wang $H$, Jin $L$, Peng $T$, Zhang $H$, Chen $Q$, Hua $Y$. Identification of cultivable bacteria in the intestinal tract of Bactrocera dorsalis from three different populations and determination of their attractive potential. Pest Manag Sci. 2014;70(1):80-7.

28. Aharon Y, Pasternak Z, Yosef MB, Behar A, Lauzon C, Yuval B, Jurkevitch E. Phylogenetic, metabolic, and taxonomic diversities shape Mediterranean fruit fly microbiotas during ontogeny. Appl Environ Microbiol. 2013;79(1): 303-13.

29. Zhao X, Zhang X, Chen Z, Wang Z, Lu Y, Cheng D. The divergence in bacterial components associated with Bactrocera dorsalis across developmental stages. Front Microbiol. 2018;9:114.

30. Denisov G, Walenz B, Halpern AL, Miller J, Axelrod N, Levy S, Sutton G. Consensus generation and variant detection by Celera assembler. Bioinformatics. 2008;24(8):1035-40.

31. Li R, Yu C, Li Y, Lam T-W, Yiu S-M, Kristiansen K, Wang J. SOAP2: an improved ultrafast tool for short read alignment. Bioinformatics. 2009;25(15): 1966-7.

32. Söhngen C, Podstawka A, Bunk B, Gleim D, Vetcininova A, Reimer LC, Ebeling C, Pendarovski C, Overmann J. Bac dive-the bacterial diversity metadatabase in 2016. Nucleic Acids Res. 2016:44(D1):D581-5.
33. Richter M, Rosselló-Móra R. Shifting the genomic gold standard for the prokaryotic species definition. Proc Natl Acad Sci U S A. 2009;106(45):1912631.

34. Pritchard L, Glover RH, Humphris S, Elphinstone JG, Toth IK. Genomics and taxonomy in diagnostics for food security: soft-rotting enterobacterial plant pathogens. Anal Methods. 2016;8(1):12-24.

35. Ciufo S, Kannan S, Sharma S, Badretdin A, Clark K, Turner S, Brover S, Schoch $\mathrm{CL}$, Kimchi A, DiCuccio M. Using average nucleotide identity to improve taxonomic assignments in prokaryotic genomes at the NCBI. Int J Syst Evol Microbiol. 2018:68(7):2386-92.

36. Emms DM, Kelly S. OrthoFinder: solving fundamental biases in whole genome comparisons dramatically improves orthogroup inference accuracy. Genome Biol. 2015;16(1):157.

37. Huerta-Cepas J, Szklarczyk D, Forslund K, Cook H, Heller D, Walter MC, Rattei T, Mende DR, Sunagawa S, Kuhn M. eggNOG 4.5: a hierarchical orthology framework with improved functional annotations for eukaryotic, prokaryotic and viral sequences. Nucleic Acids Res. 2016;44(D1):D286-93.

38. Hillis DM, Bull JJ. An empirical test of bootstrapping as a method for assessing confidence in phylogenetic analysis. Syst Biol. 1993;42(2):182-92.

39. Janda JM, Abbott SL. Bacterial identification for publication: when is enough enough? J Clin Microbiol. 2002;40(6):1887-91.

40. Foerstner KU, von Mering C, Hooper SD, Bork P. Environments shape the nucleotide composition of genomes. EMBO Rep. 2005;6(12):1208-13.

41. Wuyts S, Wittouck S, De Boeck I, Allonsius CN, Pasolli E, Segata N, Lebeer S. Large-scale phylogenomics of the lactobacillus casei group highlights taxonomic inconsistencies and reveals novel clade-associated features. mSystems. 2017;2(4):e00061-17.

42. Feng $\mathrm{H}$, Edwards $\mathrm{N}$, Anderson CM, Althaus M, Duncan RP, Hsu Y-C, Luetje CW, Price DR, Wilson AC, Thwaites DT. Trading amino acids at the aphidBuchnera symbiotic interface. Proc Natl Acad Sci U S A. 2019;116(32):1600311.

43. Dahan RA, Duncan RP, Wilson AC, Dávalos LM. Amino acid transporter expansions associated with the evolution of obligate endosymbiosis in sapfeeding insects (Hemiptera: sternorrhyncha). BMC Evol Biol. 2015;15(1):52.

44. Baumann P. Biology of bacteriocyte-associated endosymbionts of plant sapsucking insects. Annu Rev Microbiol. 2005;59:155-89.

45. Yamada R, Deshpande SA, Bruce KD, Mak EM, William W. Microbes promote amino acid harvest to rescue undernutrition in Drosophila. Cell Rep. 2015; 10(6):865-72.

46. LeBlanc JG, Milani C, De Giori GS, Sesma F, Van Sinderen D, Ventura M. Bacteria as vitamin suppliers to their host: a gut microbiota perspective. Curr Opin Biotechnol. 2013;24(2):160-8.

47. Blatch S, Meyer KW, Harrison JF. Effects of dietary folic acid level and symbiotic folate production on fitness and development in the fruit fly Drosophila melanogaster. Fly (Austin). 2010;4(4):312-9.

48. Wong AC-N, Dobson AJ, Douglas AE. Gut microbiota dictates the metabolic response of Drosophila to diet. J Exp Biol. 2014;217(11):1894-901.

49. Sannino DR, Dobson AJ, Edwards K, Angert ER, Buchon N, McFall-Ngai MJ. The Drosophila melanogaster gut microbiota provisions thiamine to its host. mBio. 2018;9(2):e00155-18.

50. Ye H, LIU JH. Population dynamics of the oriental fruit fly, Bactrocera dorsalis (Diptera: Tephritidae) in the Kunming area, southwestern China. Insect Sci. 2005;12(5):387-92.

51. Snyder AK. Rio RV: "Wigglesworthia morsitans" folate (vitamin B9) biosynthesis contributes to tsetse host fitness. Appl Environ Microbiol. 2015; 81(16):5375-86.

52. Akami M, Ren X-M, Qi X, Mansour A, Gao B, Cao S, Niu C-Y. Symbiotic bacteria motivate the foraging decision and promote fecundity and survival of Bactrocera dorsalis (Diptera: Tephritidae). BMC Microbiol. 2019;19(1):229.

53. Damodaram KJP, Ayyasamy A, Kempraj V. Commensal Bacteria aid mateselection in the fruit Fly, Bactrocera dorsalis. Microbial Ecol. 2016;72(3):725-9.

54. Itoh H, Tago K, Hayatsu M, Kikuchi Y. Detoxifying symbiosis: microbemediated detoxification of phytotoxins and pesticides in insects. Nat Prod Rep. 2018;35(5):434-54.

55. Kikuchi Y, Hayatsu M, Hosokawa T, Nagayama A, Tago K, Fukatsu T. Symbiont-mediated insecticide resistance. Proc Natl Acad Sci U S A. 2012; 109(22):8618-22.

56. Cheng D, Guo Z, Riegler M, Xi Z, Liang G, Xu Y. Gut symbiont enhances insecticide resistance in a significant pest, the oriental fruit fly Bactrocera dorsalis (Hendel). Microbiome. 2017:5(1):13. 
57. Daisley BA, Trinder M, McDowell TW, Collins SL, Sumarah MW, Reid G. Microbiota-mediated modulation of organophosphate insecticide toxicity by species-dependent interactions with lactobacilli in a Drosophila melanogaster insect model. Appl Environ Microbiol. 2018;84(9):e02820-17.

58. Yoon S-H, Ha S-M, Kwon S, Lim J, Kim Y, Seo H, Chun J. Introducing EzBioCloud: a taxonomically united database of $16 \mathrm{~S}$ rRNA gene sequences and whole-genome assemblies. Int J Syst Evol Microbiol. 2017;67(5):1613.

59. Edgar RC. MUSCLE: multiple sequence alignment with high accuracy and high throughput. Nucleic Acids Res. 2004;32(5):1792-7.

60. Kumar S, Stecher G, Li M, Knyaz C, Tamura K. MEGA X: molecular evolutionary genetics analysis across computing platforms. Mol Biol Evol. 2018;35(6):1547-9.

61. Holmes B, Willcox W, Lapage S. Identification of Enterobacteriaceae by the API 20E system. J Clin Pathol. 1978;31(1):22-30.

62. Bolger AM, Lohse M, Usadel B. Trimmomatic: a flexible trimmer for Illumina sequence data. Bioinformatics. 2014;30(15):2114-20.

63. Brooks L, Kaze M, Sistrom M. A curated, comprehensive database of plasmid sequences. Microbiol Resour Announc. 2019;8(1):e01325-18.

64. Delcher AL, Bratke KA, Powers EC, Salzberg SL. Identifying bacterial genes and endosymbiont DNA with glimmer. Bioinformatics. 2007;23(6):673-9.

65. Lowe TM, Chan PP. tRNAscan-SE on-line: integrating search and context for analysis of transfer RNA genes. Nucleic Acids Res. 2016;44(W1):W54-7.

66. Lagesen $\mathrm{K}$, Hallin $\mathrm{P}$, Rødland EA, Stærfeldt H-H, Rognes T, Ussery DW. RNAmmer: consistent and rapid annotation of ribosomal RNA genes. Nucleic Acids Res. 2007;35(9):3100-8.

67. Nawrocki EP, Burge SW, Bateman A, Daub J, Eberhardt RY, Eddy SR, Floden EW, Gardner PP, Jones TA, Tate J. Rfam 12.0: updates to the RNA families database. Nucleic Acids Res. 2015;43(D1):D130-7.

68. Benson G. Tandem repeats finder: a program to analyze DNA sequences. Nucleic Acids Res. 1999;27(2):573-80.

69. Hasan MS, Liu Q, Wang H, Fazekas J, Chen B, Che D. GIST: genomic island suite of tools for predicting genomic islands in genomic sequences. Bioinformatics. 2012;8(4):203.

70. Zhou Y, Liang Y, Lynch KH, Dennis JJ, Wishart DS. PHAST: a fast phage search tool. Nucleic Acids Res. 2011;39(suppl_2):W347-52.

71. Grissa I, Vergnaud G, Pourcel C. CRISPRFinder: a web tool to identify clustered regularly interspaced short palindromic repeats. Nucleic Acids Res. 2007;35(suppl_2):W52-7.

72. Kanehisa M, Furumichi M, Tanabe M, Sato Y, Morishima K. KEGG: new perspectives on genomes, pathways, diseases and drugs. Nucleic Acids Res. 2017;45(D1):D353-61.

73. Galperin MY, Makarova KS, Wolf Yl, Koonin EV. Expanded microbial genome coverage and improved protein family annotation in the COG database. Nucleic Acids Res. 2015;43(D1):D261-9.

74. O'Leary NA, Wright MW, Brister JR, Ciufo S, Haddad D, McVeigh R, Rajput B, Robbertse B, Smith-White B, Ako-Adjei D. Reference sequence (RefSeq) database at NCBI: current status, taxonomic expansion, and functional annotation. Nucleic Acids Res. 2016;44(D1):D733-45.

75. Boeckmann B, Bairoch A, Apweiler R, Blatter M-C, Estreicher A, Gasteiger E, Martin MJ, Michoud K, O'Donovan C, Phan I. The SWISS-PROT protein knowledgebase and its supplement TrEMBL in 2003. Nucleic Acids Res. 2003;31(1):365-70.

76. research $\mathrm{GOC}$. The Gene Ontology $(\mathrm{GO})$ database and informatics resource. Nucleic Acids Res. 2004;32(suppl_1):D258-61.

77. Chen L, Yang J, Yu J, Yao Z, Sun L, Shen Y, Jin Q. VFDB: a reference database for bacterial virulence factors. Nucleic Acids Res. 2005;33(suppl_1): D325-8.

78. Liu B, Pop M. ARDB — antibiotic resistance genes database. Nucleic Acids Res. 2009;37(suppl_1):D443-7.

79. Urban M, Cuzick A, Rutherford K, Irvine A, Pedro H, Pant R, Sadanadan V, Khamari L, Billal S, Mohanty S. PHI-base: a new interface and further additions for the multi-species pathogen-host interactions database. Nucleic Acids Res. 2017:45(D1):D604-10.

80. Lombard V, Golaconda Ramulu H, Drula E, Coutinho PM, Henrissat B. The carbohydrate-active enzymes database (CAZy) in 2013. Nucleic Acids Res. 2014;42(D1):D490-5.

81. Jehl M-A, Arnold R, Rattei T. Effective-a database of predicted secreted bacterial proteins. Nucleic Acids Res. 2010;39(suppl_1):D591-5.

82. Gurevich A, Saveliev V, Vyahhi N, Tesler G. QUAST: quality assessment tool for genome assemblies. Bioinformatics. 2013;29(8):1072-5.
83. Parks DH, Imelfort M, Skennerton CT, Hugenholtz P, Tyson GW. CheckM: assessing the quality of microbial genomes recovered from isolates, single cells, and metagenomes. Genome Res. 2015;25(7):1043-55.

84. Seemann T. Prokka: rapid prokaryotic genome annotation. Bioinformatics. 2014;30(14):2068-9.

85. Page AJ, Cummins CA, Hunt M, Wong VK, Reuter S, Holden MT, Fookes M, Falush D, Keane JA, Parkhill J. Roary: rapid large-scale prokaryote pan genome analysis. Bioinformatics. 2015;31(22):3691-3.

86. Price MN, Dehal PS, Arkin AP. FastTree 2 - approximately maximumlikelihood trees for large alignments. PLoS ONE. 2010;5(3):e9490.

87. Buchfink B, Xie C, Huson DH. Fast and sensitive protein alignment using DIAMOND. Nat Methods. 2015;12(1):59.

88. Darzi Y, Letunic I, Bork P, Yamada T. iPath3. 0: interactive pathways explorer v3. Nucleic Acids Res. 2018;46(W1):W510-3.

\section{Publisher's Note}

Springer Nature remains neutral with regard to jurisdictional claims in published maps and institutional affiliations.

\section{Ready to submit your research? Choose BMC and benefit from:}

- fast, convenient online submission

- thorough peer review by experienced researchers in your field

- rapid publication on acceptance

- support for research data, including large and complex data types

- gold Open Access which fosters wider collaboration and increased citations

- maximum visibility for your research: over $100 \mathrm{M}$ website views per year

At BMC, research is always in progress.

Learn more biomedcentral.com/submissions 